\title{
Navigating the Virtual Landscape: Co-ordinating the Shared Use of Space
}

\author{
Phillip Jeffrey \\ Department of Computing \\ \& Information Science \\ University of Guelph \\ Guelph, Ontario Canada N1G 2W1 \\ Phillip.Jeffrey@acm.org
}

\author{
Gloria Mark \\ GMD-FIT \\ German National Research Center for \\ Information Technology \\ 53754 Sankt Augustin, Germany \\ Gloria.Mark@gmd.de
}

\begin{abstract}
Collaborative Virtual Environments, such as multi-user domains MUDs, chatrooms, or three-dimensional graphical environments, provide a common space for people to interact in, independent of geographical location. In this chapter, we examined how the different metaphors used to represent two-dimensional and three-dimensional environments might influence interpersonal behaviours. We focused on behaviours related to navigation and positioning: 1) proxemics, the maintenance of personal space, 2) the signaling of private space, and 3) the effects of crowding. We discovered that the design of the three-dimensional space offer sociopetal spaces that encourage interaction, make clusters of actors easily visible, and provide cues so that people maintain a sense of personal space. In both environments, adverse reactions to crowding occurred. We suggest that differences in interpersonal behaviours may be influenced by an embodiment (avatar), design features of the space, and the number of other actors present. In a three-dimensional environment, these factors appear to influence navigation and positioning in the environment.
\end{abstract}

\section{Introduction}

Collaborative Virtual Environments (CVEs) have been attracting interest for their potential to support communication, collaboration and co-ordination of groups. Already we are seeing cases where such environments are proving effective as shared information spaces for collaborative tasks e.g. multi-user domains (MUDs) [1] and graphical virtual worlds [2]. A special case of CVEs employ a three-dimensional graphical representation of space in which users are represented as a figure (i.e. avatar). Examples of diverse approaches to these types of CVE designs includes the DIVE system [3]; MASSIVE [4]; and Active Worlds [5].

The advantage of using such systems is that group members located in different geographical places can therefore occupy the same virtual space, and thus have a common frame of reference for collaboration. Many CVEs, whether text-based or graphical, are synchronous, which offer actors the advantage of receiving immediate feedback; one can present one's position, field questions, and receive immediate answers. Such immediate feedback is useful for clarification of an issue and, it has been argued, may lead to group cohesiveness [6]. Users' interaction and navigation within a virtual space may be influenced by a variety of factors, among them, how the space is designed, the presence of other users, and the positioning of artefacts. This result was found in an empirical study involving rearranging artefacts in a virtual CVE room; the choice of targets was to some extent determined by other participants' behaviours [7].

The purpose of this paper is to examine the question of how a three-dimensional representation of space in a CVE might, if at all, influence interaction among the participants. To answer this question, we observed interaction in two CVE's distinguished by how their space is represented; we contrasted interaction in a text-based chat environment that employs a three-dimensional representation of space, with a purely textbased chat environment. In this exploratory study we were particularly interested in how the design of space and the presence of others affect navigation and positioning. Our selection of interpersonal behaviours to observe was motivated by choosing those that concern spatial positioning and navigation among actors. This led us to choose behaviours that show how personal space is maintained, how privacy is indicated, and reactions to crowding. In the following, we provide a framework in which to understand the relevance of such types of social interaction in a CVE. 


\section{Shared Information Spaces}

A CVE is a kind of shared information space. Bannon and Bødker [8] describe that shared information spaces are characterised not only by the information present, but also on the interpretation and the meaning derived from it by the users that inhabit the environment. A digital library, chatroom, or even graphical virtual world must provide a meaningful context for collaborative and co-operative work. We view an information space as an environment such as the World Wide Web (WWW) that presents information to be interpreted to the user and that can be extracted or previewed based on how one navigates through the space. Enhancements to CVEs already enable the capability to link to Web pages [e.g. 9], shared virtual workspaces [10], and even physical places [11].

\subsection{Spatial metaphors and connotations}

How the information is found and interpreted in such a space is important. Spatial metaphors have been shown to be valuable devices to aid navigation in virtual environments. The experience of the WWW has been described using the metaphor of a mansion, filled with rooms, continually expanding [12]. People have been found to use spatial metaphors while navigating the WWW for information, perceiving it as a landscape that they move through physically, or as navigation towards information [13]. These same authors found in a later study that experienced and inexperienced users differed in their descriptions of their web experience [14]. Both experts and novices used language representing physical movements and actions towards information (e.g. "go", "went") rather than simply passively receiving it. However, when language usage involved trajectory movement, experts perceived themselves as an active agent, navigating through the information space (active) (i.e. "I went into this thing called Yahoo"). In contrast, beginners were more likely to see the web as the agent, with the information moving toward them (i.e. "It brought me to the Anthropology page"). Maglio and Matlock [14] suggest that these results indicate that WWW users view the virtual space within a familiar context of a physical space.

Other metaphors used in CVEs include a city metaphor applied in a MUD environment [15] and the results suggest that spatial metaphors can be helpful tools for navigation. Some chatroom environments use metaphors from the physical world such as a house or extended hallway, with different rooms having different functions or purpose. A "homepage" represents a personal location on the WWW, further reinforced with its location marker as an "address". Language associated with the WWW such as "surfing", "explorer", and "navigator", and with CVEs such as "teleporting" all convey the notion of movement and traversal through a virtual landscape. In [16], Dieberger describes navigation in the information spaces of graphical user interfaces and WWW hypertext in terms of "magic features" which provide shortcuts for increased efficiency. If the source of a metaphor is something in the physical world, then features such as teleportation that disrupt this metaphor may be seen to originate from a magical world.

The contextual cues of a place may contribute to suggesting appropriate behaviour within that space. Dieberger [17] refers to these cues as social connotations. Certain behaviours that may be appropriate within the privacy of one's bedroom are forbidden in a public workplace. In addition, the nature of navigation may also be influenced by the social connotation conveyed by a place [17]. The same space in fact may connote different interpretations of appropriate behaviour at different occasions, such as a community hall that may be used for a music concert, a wedding or for a local sports event. Spaces that don't provide what Harrison and Dourish [18] refer to as a sense of place may adversely affect conversations and the participants' behaviour. Thus, it appears that the design of a CVE, and the cues it contains, can influence the nature of the interaction and movement through that space.

This idea is intuitively obvious when we think of physical environments, such as workplaces, which have different spatial layouts. Architects and interior designers have long been concerned with the spatial positioning of offices, desks and public areas in designing environments for work. Not only can the interior layout influence the degree of interaction between employees, but it can also define the hierarchy within the workplace, and distinguish areas for work from those for socialisation. Even the placement of shared artefacts is crucial since they can provide peripheral awareness information about other people's activities [19]. Osmond [20] classifies space as being sociopetal or sociofugal. Sociopetal spaces (i.e. a cafeteria) encourage interpersonal communication. Sociofugal spaces, such as a lobby waiting area, are designed to 
restrict or discourage social interaction. A working environment where people perceive the space as sociopetal should have expectations that social communication will take place.

Although we may exist in space, we actually refer to artefacts and architecture within the context of a place. Tuan [21] believes that we navigate through space until we pause, at which point an awareness of our positioning with respect to artefacts transforms our location into a place. This perception is similar to that proposed by Altman and Zube [22] who state that as individuals become more familiar with a space, they learn to associate meaning with it, and thus through experience, a space is perceived as a place.

\subsection{Social navigation}

It may be that the perception of a space as a place is facilitated as interacting partners develop a shared understanding of the environment [17]; this creates a dynamic process as interaction within a space fosters meaning. Social navigation has been described as navigation through collected information that is enabled due to the activity of others. Dieberger classifies social navigation as being direct or indirect [17]. Direct social navigation involves active and direct interaction between users such as pointing out information required. Indirect social navigation is more passive and may involve recognising and identifying navigational cues left by users found in the information space.

In our definition, we believe that navigation within an information space such as a virtual environment could be considered "social navigation" when the presence of others within the shared space influence one's direction of movement or choice of position. Social navigation involves an awareness of other users who are currently present or have been there in the past. This may involve the process of recognition of others; cues such as a username or unique personal identifiers signal familiarity. The user who is navigating within the environment is completing a goal-oriented task -- finding others to interact or seeking information from others. This view is similar to that proposed by Dourish and Chalmers ([discussed in 18]) who describe that clusters of individuals serve as focal points where people navigate to. The type of environment may determine the type of questions asked, the behaviour that is appropriate, and how users will respond [17].

Many virtual environments are designed as spaces primarily intended for socialisation and interaction; in this context, navigation serves to find others. In the physical world, public spaces such as local pubs or neighbourhood parks are used as areas for meeting new people and reacquainting relationships with friends. Similar places may also be provided within virtual space. Thus, the process of socialisation with others in a shared space may involve both communicative as well as navigation behaviours.

\subsection{Social norms for shared spaces}

Thus far we have discussed the notion of how one's perception of and behaviour in a shared information space is affected by the presence of others. As the level of interaction increases, shared norms, values and expectations emerge in a group. Expectations about behaviour during interpersonal interaction exist as social guidelines. This is to ensure that group members behave in a regulated manner as participants within the group specifically, and more broadly, as members within an organisation or society [23]. Shared expectations of behaviour also have sanctions, which lead to uniformity in the behaviour of group members. These standards of behaviour can be commonly referred to as norms [24].

Group norms are informal regulations that are usually unwritten and implied [25]. They have two main purposes: to create a frame of reference for understanding within the group, and to identify appropriate and inappropriate behaviour [26]. Norms are not static but rather dynamic. Determinants such as the environment, one's culture, and the composition of the group will influence the emergence, acceptance and effectiveness of norms by the members [27]. The existence of group norms is an important part in helping members feel a sense of integration within the group.

Carry-over behaviour, according to Feldman [25], is one method that enables social conventions to develop within a workgroup. Individuals, through past experience as participants in other groups or similar working environments, are believed to transfer those previously learned conventions into their current group. Violations of social norms may produce conflict between the violator and the other group members, 
increase the level of interpersonal communication directed at the violator about appropriate behaviour, or result in dismissal from the group [24].

Thus, social norms regulate behaviour within society as well as one's social group and play an important role in one's own behaviour as well as expectations about the behaviour of others. The members of a workgroup or social group have dual roles as societal members in the physical environment. Therefore, it is expected that conventions are not only transferred from group to group, but also are transferred from society to a group. It seems reasonable then that interpersonal behaviours and social conventions might transfer from experiences with the physical use of shared space to a virtual shared space. In fact we may view CVEs as "social spaces" as an awareness of other users may produce conditions that foster social conventions in order to regulate interactions. Virtual environments are still a novelty compared with faceto-face interaction. With any technological invention, users develop new usage conventions that are appropriate for the new technology by transferring over and modifying familiar metaphors [28]. However, familiar norms such as when to answer a telephone call must be modified when using mobile phones, due to the often public nature of such conversations. Dix et al., [29] suggest that success with a new media environment may be dependent on the successfulness of transferring these norms.

Behavioural research on social interaction in CVEs has suggested that social conventions are formed and used as common communication systems in text-based MOOs [30, 17, 31], newsgroups [32], as well as in graphical environments $[33,34,35]$. In the following, we introduce a set of interpersonal behaviours which people use to coordinate interaction in physical shared spaces.

\section{Personal Space}

Proxemics is the study of personal space, a field founded by Edward T. Hall [36]. It focuses on the societal use of space to attain comfortable conversational distances and obtain preferred levels of interpersonal involvement. The study of proxemics focuses on theories related to the distances expressed during social interaction. In this sense individuals are regarded as active participants within their environment, rather than passive observers. Personal space may be defined as an area with invisible boundaries surrounding an individual's body which functions as a comfort zone during interpersonal communication [37, 38]. Violations may result in adverse and emotional reactions [39].

According to the principle of proximity, individuals who are physically closer develop a stronger attraction to each other than when they are further apart [26]. According to Hall [36], one's preferred distance for comfortable communication has societal (contact vs non-contact), social (stranger vs friend) and conversational intimacy (business vs casual) determinants. Face-to-face communication is often seen as the ideal setting that computer-mediated forms of communication strive to emulate [29]. Therefore, the question of whether a sense of personal space might also exist in CVEs, as it does in face-to-face interaction, was examined, keeping in mind that a sense of personal space in a CVE may be a function of the nature of the embodiment and degree of immersive experience.

\section{Private space}

In social interaction, privacy is the selective control by individuals or groups of personal information, the degree of interpersonal communication, and the level of social interaction [40, 41]. An imbalance results when one's perceived level of social interaction differs from one's optimal level. The need for group and individual privacy and its related social conventions are common throughout physical world societies, with studies showing that the preference for privacy, disclosure or social interaction is cultural and situationdependent [41]. This desire for private spaces for interaction is reflected in the design of CVEs, which provide password-protected private rooms or whisper commands, which create a private, shared communication space. On this topic, we investigated how behaviours indicated a desire for a private space in a CVE.

\section{Crowding}

Crowding is a psychological perception characterised by feelings of personal space violations if one's current level of social interaction is higher than preferred [40]. Stokols [42] refers to social crowding as 
feelings of being crowded due to the presence or awareness of others, which can lead to stress. However, stress due to crowding was found to be lower when individuals received positive group feedback [43] and where groups were in an atmosphere of getting to know each other rather than evaluating one another [44].

The feeling of crowding in virtual environments has been intentionally simulated to help people overcome phobias [45] which suggests that such a simulation can provoke genuine feelings of being crowded by others. A technical solution to support crowds in CVEs has been implemented in MASSIVE-2 [46], although the psychological effects have not been examined. We examined here how users react to crowding in the CVEs that we chose.

The commonalities linking personal space, private space and crowding are related to people's shared use of space. Each of these social norms involves physical spaces that may be perceived as personal although interaction occurs within public environments. We now examine these behaviours within virtual space.

\section{Methodological Approach and Research Setting}

To examine the relationship of users in shared virtual spaces, we have explored two contrasting online, virtual environments: Active Worlds ${ }^{1}$ (AW) (http://www.activeworlds.com) and WebChat ${ }^{2}$ (WBS) (http://wbs.net) in order to examine social conventions that may exist through one's interaction and navigation. All environments are multi-user and are accessible from the Internet. The environments differ in terms of their means for navigation and their representation in space, which enabled us to detect differences that might be due to the design of space.

WBS is designed in a flat two-dimensional space using the spatial metaphor of a house, each with different "rooms" serving as a different chat environment (Fig. 1). Together the rooms form what is called "a community". Communication is text-based.

AW is designed along the spatial metaphor of a three-dimensional physical world or landscape. Navigation occurs within and between "worlds" where users communicate with text and use graphical representations (i.e. avatars) (Fig. 3).

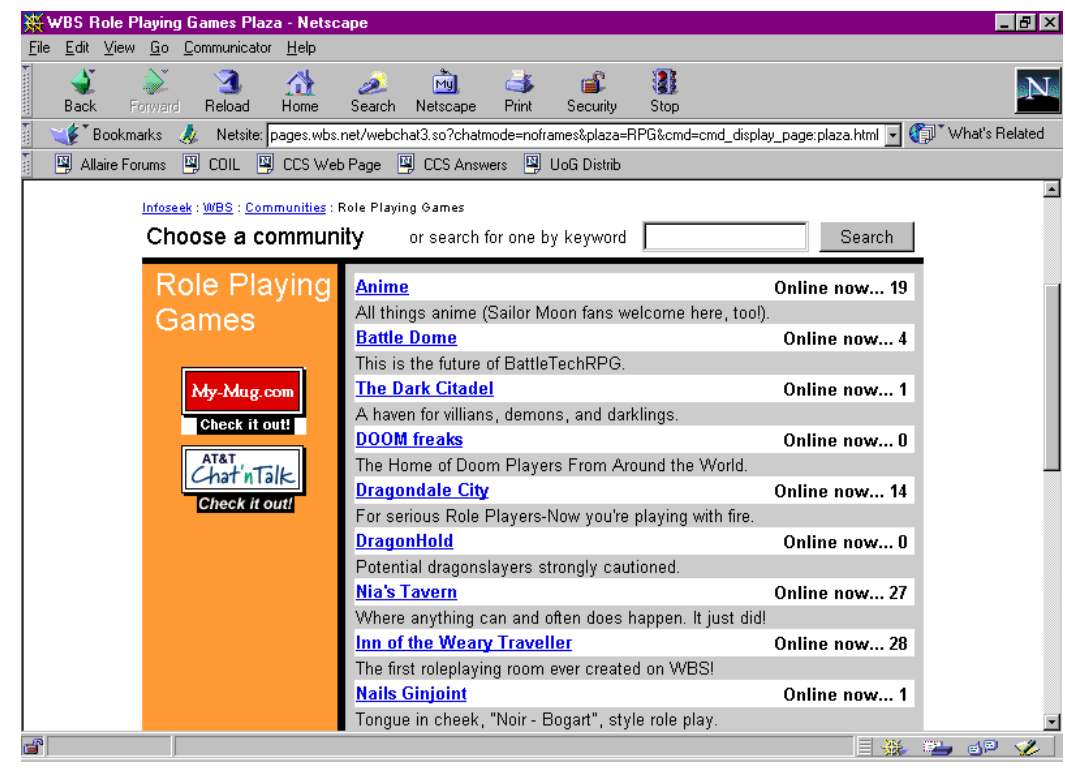

Fig. 1. WBS: Role Playing Games Chatrooms

\footnotetext{
${ }^{1}$ Copyright $(C) 1995$ - 1998 Circle of Fire, Inc., http://www.activeworlds.com.

${ }^{2}$ Copyright (C) 1995 - 1998 Infoseek Corporation., Inc, http://wbs.net
} 
The basic functionality available for navigation, communication and representation in these virtual environments is:

- WBS: communication is text-based into an input box. All public messages appear in a large text-area and individuals can alter their size and colour. Individuals are represented with user names and can attach pictures, images or WWW links to messages. Private messages can be sent using an internal email message system or directly to the desired user during conversation. Private rooms can be created for private conversations. Socialising occurs within a room and individuals can easily navigate within and between rooms using the mouse.

- AW: full-bodied avatars can walk and exhibit movements dependent on the particular world visiting and one's membership status (i.e. citizen or tourist). Possible movements include waving, dancing, and fighting activated by mouseclicks. Avatars can navigate in three dimensions by using the arrow keys or the mouse. Communication is textbased from the keyboard. All public messages appear in a scrollable window and may also appear above the avatar's head with the avatar's name for 30 seconds or until the next typed message appears, if this functionality is desired. Citizens can send private messages by clicking on the telegram icon to access the internal message system.

Approximately 50 hours of time was spent observing the two different online environments. The observations were performed in a period spanning September 1997 through November 1998, although not continuous. Online recording occurred in AW in order to ensure accurate data.

We were interested to what extent notions of space in the physical environment might transfer into a threedimensional graphical virtual environment, and whether references to these notions also appear in a textbased chat environment. We coded the following behaviours: 1) Is a certain personal distance kept? 2) What happens when this distance is violated? 3) When people navigate through the environment, do they disturb others' personal space? 4) Does personal space exist in a primarily textual environment? 5) How do people express privacy? 6) How do people respond to environments that they perceive are crowded?

\section{Observations}

In comparing our observations, we find differences in how the social behaviours we examined are expressed within each virtual space. In each environment, such behaviours appear to be influenced by the available functionality for representation and communication. We describe our observations as follows.

\subsection{Personal Space: Social Positioning}

In the text environment of WBS, instances were rarely observed that indicate that users behaved as though they had a sense of personal space. The following is one example that occurred in a role-playing chatroom called "Inn of the Weary Traveller".

Steven: *edges closer and closer to DAYNA [another user] until within talking distance ${ }^{*}$ hello... * Grin* 


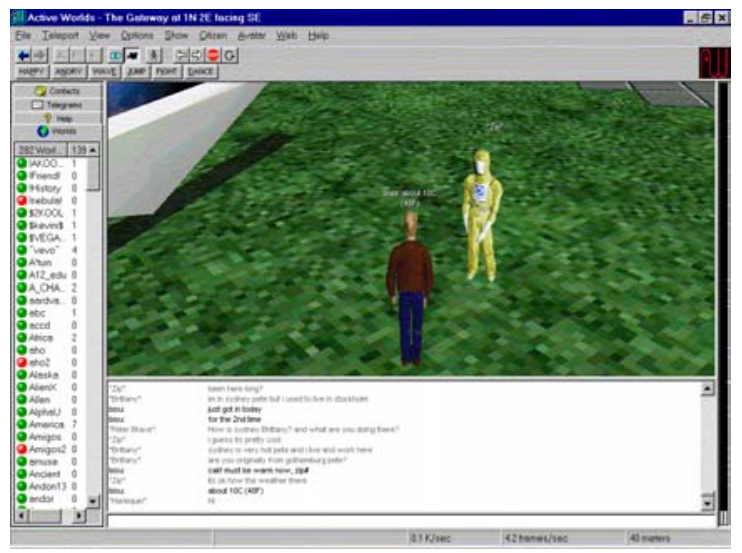

Fig. 2a. Comfortable personal distance

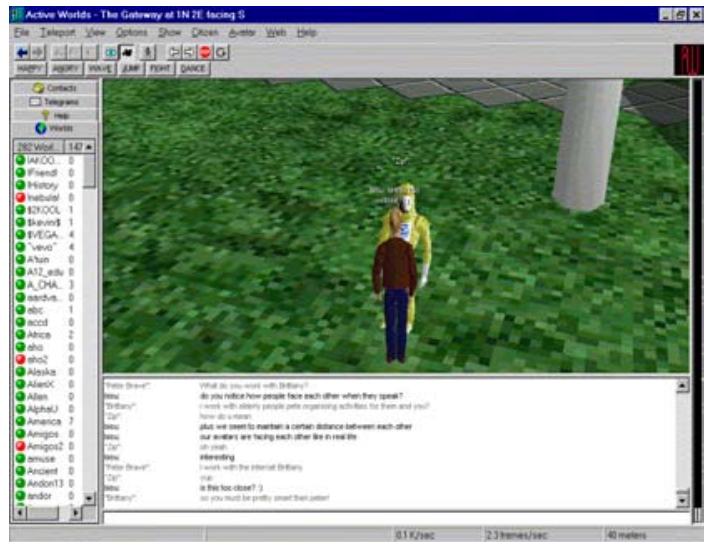

Fig. 2b. Personal space invasion

In contrast, behaviours involving personal space were regularly observed in AW. During interpersonal communication, avatars maintained a distinct personal distance. In areas with open spaces, such as the landing point in each world, which also functions as a common meeting place, the avatars of a number of users were present. Invasions of personal space occurred when the Observer $(\mathbf{O})$ moved the avatar from a comfortable personal distance and positioned it face-to-face close to another [Fig. 2a and Fig. 2b]. Reactions to personal space invasions were generally expressed through verbal or non-verbal behaviour and were observed to signal discomfort, as in the following example.

O met Laracat in the AlphaWorld supply dock getting building materials. O moved faceto-face and immediately Laracat moved back maintaining a slightly larger distance than had been previously observed and remarked:

Laracat: actually...It's funny...but it does make me uncomfortable when another avatar gets "too close" in my avatar's face.

O: [moves forward] why?

Laracat: [moves back] just does

Laracat: This is a nice distance to keep.....)

Laracat: [I backed up] the same way I'd back up if a "real" person got up that close....someone I don't know very well

On several occasions in $\mathrm{AW}, \mathrm{O}$ experienced a personal space invasion. When $\mathrm{O}$ was teaching a user named "trooper" to fly, "trooper" passed through O's avatar and then apologised.

"trooper": sorry about that...you sure do speed up with the ctrl key! :-)

Having an avatar navigate into one's personal space was unappreciated, as the comments below suggest:

Wepwawet: something about personal space,...we carry the conventions of [real life] into $A W$

22much:... i need my personal space here

Geophrey: Netiquette will be amended to include "personal space" in [virtual reality] I'm sure :-)

Dizzy: I try to walk around [avatars]. Courtesy you know.

Danaidae: [avatars] need [their] personal space too

Blade:...it feels wrong/strange like we stare at each other if we are [too] close don't you think?

Thus, we observed that within the three-dimensional graphical environment of $\mathrm{AW}$, individuals preferred to maintain a comfortable personal distance during conversation. When the $\mathbf{O}$ removed the personal distance and moved face-to-face in close proximity to another avatar, behavioural reactions indicated that the other participants realised their personal space had been violated. Individuals preferred to navigate around rather than through other avatars.

As AW uses a metaphor of three-dimensional space, it may then produce a place conducive for behavioural conventions such as personal space to emerge. The AW observations parallel those found in physical shared spaces. Physical proximity plays a significant role in how workgroups communicate, collaborate and co-ordinate tasks [47]. In face-to-face interpersonal communication, people change their positioning to 
maintain their personal space [36], and use non-verbal communication such as gestures and turn-taking for assistance in regulating conversation [48]. Reactions to personal space invasions have been perceived in mediated environments as threatening $[49,50]$.

There may also be a practical reason for adverse reactions to personal space invasions in AW. Participants used the first person perspective for communication and viewing the immediate surroundings. During faceto-face personal space invasions, one's viewpoint is obscured if using the first person perspective. This may explain negative reactions towards personal space invasions, namely, that standing in front of an avatar will block the viewpoint, similar to real life. Personal space may then affect people differently depending on their avatar perspective (i.e. first or third) or where their vision is primarily focused (i.e. viewscreen or text window).

Geophrey: ... when you stand in someone's face, you're taking up most of their viewing area ;-)

We attribute the low referral to spatial behaviour in WBS to the text-based nature of the system. This behaviour may be due to the lack of a distinct spatial location provided for participants of textual environments such as chatrooms or Multi-User Domains (MUDs) [51]. A perception of personal space may also be due to the result of avatar embodiment. What it may mean is that personal space, as a nonverbal cue for communication, may not have an equivalent in a purely textual environment such as WBS. According to Fletcher [52], nonverbal behaviours such as personal space and gestures complement one's communicative message and are ineffectively transferred in computer-mediated communication such as email, the Internet or chat groups. This should not be surprising. In MUD studies of non-verbal communication $[53,54]$ personal space seems to be redefined in terms of private places rather than the proxemic definition defined by Hall [36] for physical world spaces. Therefore, it may be that personal space does in fact exist in text-based WBS, when we re-define it in terms of private areas of the metaphorical rooms.

\subsection{Private space: In the world but not of it}

In WBS, messages are typed into a chat-input window and appear immediately on the message screen. Functionality exists to send private messages and images to other users instantaneously, to leave messages in users' personal WBS email box, or to create a private room. Often more people were present than those participating; this could be due to using ICQ, a private real-time chatting system simultaneously with WBS, or they may have been passive observers rather than active participants. Sometimes people would express in their tagline attached to their message, or through a public message, whether private messages are desired, as the following user's tagline indicated.
Lizabeth21 (no PMs [private messages] please!!!):
I dislike private messages. Because there's nothing that anyone can say to me that can't be said in public
Lizabeth21: $\quad$ Plus in my experience, I only get nasty messages in PM ...not many have the guts to be crude, rude, crass in public

Similar to WBS, functionality in AW exists for citizens to send private messages to each other but avatar positioning sometimes also indicated that a conversation was private. Avatars were observed positioned high above others watching the avatars below or two avatars were positioned face-to-face in close proximity. Other examples that may also be indications of privacy markers include avatars that were observed separated from the main gathering points either above ground or on the same plane. Attempts at communication usually resulted in non-responses or the avatars leaving to another location.

Our observations indicate that a desire for private spaces exists in virtual environments. In both environments, people used functionality to engage in private conversations and to create privacy markers. Westin [41] classifies privacy into four different definitions: solitude, intimacy, reserve and anonymity. Solitude and intimacy may be most relevant to the environments observed. Westin defines solitude as choosing to separate oneself from observation by other people. In both environments this behaviour, referred to in CVE vernacular as "lurking", occurred as individuals observed the social interaction of others without participating themselves. In WBS, this was noticeable, as the population of the world was usually higher than the number of active participants:

$$
\text { vista-958: } \quad \text { Thirty-five people in here and only } 15 \text { are talking }
$$


Intimacy is commonly associated with close knit friends or small workgroups. Personal messages in WBS and telegrams in AW provided a means for more intimate conversations. In AW, dyads and groups were observed face-to-face sometimes during conversation, creating, perhaps, a privacy marker by their avatar positioning. Thus people create private spaces in three-dimensional environments.

One reason for this privacy behaviour that we observed may be that people, especially new users, are trying to understand appropriate behaviours from watching others. By removing oneself from the main activity centre, one can become an observer, without the pressure to interact with others.

\subsection{Crowding: Navigation and design constraints}

In each environment there was a limit on the number of users that could comfortably communicate, and in AW, navigate. Reaching these upper limits created technical and social problems. If the environment is perceived as crowded, individuals have a number of options available. They could remove themselves from the environment, limit the numbers of conversational partners in order to minimise the number of messages missed, or use direct, private methods of communication such as telegrams or personal messages.

Indicators in WBS list the most populated public rooms that can be immediately accessed. Communities are listed by current population, e.g. Thirtysomething, 61, Inn of the Weary Traveller, 26. Communication is often disrupted due to crowding. Crowding is not only related to the number of people but also the size of messages or pictures that are sent. When there are too many people present in a room the server may be affected causing messages to first stall and then to rapidly speed past. This requires extra effort for the user who must scroll back to read missed messages.

In other instances, a large number of users made it difficult to be "heard". In a WBS chatroom with 137 users present, $\mathrm{O}$ repeatingly tried to initiate conversation with others present for 45 minutes without one reply. In both WBS and AW, individuals expressed displeasure when ignored, such as:

SneakinSam: $\quad i$ dont know what it is, but it happens all the time.... WHY IN THE HECK DOES

EVERYONE IGNORE ME?! oh well, i'll never know, adios

In AW, users must not only attend to the flow of the text as in WBS, but must also be aware of the positioning of other avatars. Functionality exists that enable people to communicate with either the 12 or 50 nearest avatars. A large number of avatars concentrated in one's immediate vicinity led to a number of observable difficulties. The ability to effectively navigate without violating the personal space of others decreases significantly as the density of avatars increases. Again, it is extra effort for the users, some of whom report strategies on where to focus their attention:

Lixx Array: $\quad$ it's a two way thing for me...either chatting, or exploring/building...where my focus on the screen is

Geophrey: $\quad$ When it's slow like it is now the chat window can be narrower. But when it gets busy I stretch so there's only a sliver for viewing.

When text was displayed above the head of each avatar (an option), then crowding of avatars made the text difficult to read since it overlapped. Monitoring one's own conversations, understanding the thread of other conversations, or maintaining conversations with more than one person became quite difficult with crowding. Concerning the central interaction area, one user expressed that she never stays there since it is too crowded.

In WBS, pictures that consumed a large area of the screen would cause difficulties for some users whose computers were unable to handle the increased bandwidth. In AW, some individuals would maximise the text screen and minimise the viewer screen in order to view more messages at one time in crowded environments.

The effect of crowding, a type of information overload, may have cognitive, technical, and visual implications. Messages streaming too fast are difficult to read; it is easy to miss personal messages. In both virtual worlds, a high volume of users and messages combined with a low baud modem or slow Internet browser resulted in a system slowdown or users being disconnected. This disrupted social interaction as 
users must spend a few minutes re-entering the system, placing a "booted" message on the screen, and catching up on the messages that were missed. Therefore, crowding made both navigation and communication within the environment more difficult:

Darrs:

Bob don't know how those people talk in those crowded rooms

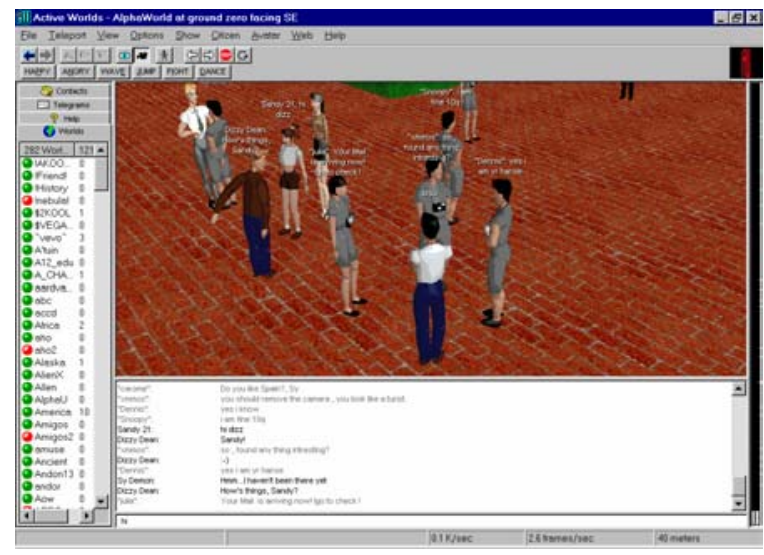

Fig. 3. Group conversation in AW

\subsection{Group space: Positioning and movement within a group}

In WBS, group membership was indicated by a common thread of conversation. Similarly in AW, group membership was also indicated through common threads of conversation as well as sometimes the positioning of avatars (Fig. 3). However, it was also observed that moving together or using the same motions conveyed group membership in the environment, e.g. simultaneously flying up and down in rhythm (i.e. bobbing vertically), walking together, dancing, or teleporting together. Such simultaneous movements served to create a weak "social boundary", separating this group from others.

Common language also created a form of social boundary. In AW, when $\mathrm{O}$ was speaking to Stine, a native Norwegian speaker, another disrupted the conversation by speaking to Stine in their native language. The shape of the group changed from a dyad to a triad, although $\mathrm{O}$ could not participate in the Norwegian conversation.

The face-to-face positioning of avatars indicated visually a group formation. We observed also in AW that when the avatars were positioned face-to-face, the relative distance between them would increase as group size increased. The physical shape was determined by group size: two avatars formed a line; three, a triangle; four, a diamond; and larger groups adopted a circle-shaped form. As additional members navigated into a group, group members would reposition, expanding the social space and relative personal space of the group.

Was this lack of embodiment in WBS compensated for in any way by the text medium? The answer seems to be that to some extent, users tried to portray embodiment through text. For example, participants created a form of virtual embodiment using self-portraits, filling out their user profile, or attaching links to homepages. And as mentioned, in role-playing chatrooms, users took on identities consistent with the themes.

\subsection{Movement through space}

In each virtual environment, users must first determine which particular world to enter. In WBS, worlds are grouped into categories, which contain related themes. Either one navigates through categories and themes to reach a room, e.g: Destinations \& Travel $\rightarrow$ Europe, or the user navigates directly to a community using a menu command. Users can also navigate room to room. Individuals sometimes would leave the room temporarily to navigate to another's homepage or to temporarily view pictures sent to them. 
Although seldom occurring in WBS, using text to describe navigation occurred occasionally in role playing rooms. For example, in the WBS "Star Trek: Nexus Bar", text descriptions which were encased in asterisks indicated navigation, thoughts, and gestures.

Lady Milldorf2: ...decides to leave the bar *walks along the wall towards the door*

In $\mathrm{AW}$, in contrast to using the first person during communication and when viewing the surrounding area, third person was used more for navigation and situations where it was important to see one's avatar, e.g.:

"trooper": $\quad$ talking is better in $1^{\text {st }}$ and moving is better $3^{\text {rd }}(-)$

Danaidae: $\quad$ only use third when checking out avatars or trying to position myself precisely

Seeing oneself during navigation helps minimise personal space and group space violations of other avatars and provides a better view of what personal and group space boundaries are. In other words, it enables one to navigate around invisible boundaries rather than pass through other avatars. From user comments, it appears that they see this as impolite behaviour, and in fact a violation of personal space.

\section{Summary and Conclusions: Co-ordinating the Shared Use of Space}

Virtual information spaces are being designed along physical world spatial metaphors: e.g. digital libraries [55], CVEs [56], and information spaces such as StackSpace and InfraSpace [57]. They enable the user to visualise information and can provide an immersive environment for collaboration of work activities as physical objects are represented as virtual artefacts, as people become virtual embodiments and representations, and as workplaces become virtual landscapes. A key to the effective use of these spaces should therefore be that the information space is designed to be both meaningful and logically organised for the task.

Familiar spatial concepts may not be sufficient to guide navigation within these new forms of shared information spaces, even though they are found to be used $[12,13,14,15]$. Consistent with the spatial metaphor of a physical world, users walk through AW. However, the ability to teleport within and between worlds may represent navigation that deconstructs this spatial metaphor [16]. In WBS, it can also be argued that the teleport functionality in chatrooms, and the ability to navigate through the WWW and back is also inconsistent with a spatial layout of rooms.

The design of the two-dimensional and three-dimensional CVE spaces, in our opinion, helped guide the nature of the interaction. Navigation in WBS occurred mostly between chatrooms and the WWW rather than within a particular chat community. Information artefacts, such as user profile icons, contain selective personal information which enable others to immediately access and navigate to someone's personal home page or send email. The degree to which this information was meaningful and navigable may have assisted users in developing a mental image of others that enriched conversation and facilitated social interaction. We observed users coming back after visiting another user's homepage or requesting others to accompany them to another room. Navigation often relied on the assistance of others, suggesting it was a collaborative effort [57].

The design of the virtual space in AW also influenced interaction by offering sociopetal spaces conducive for interaction [20]. In most worlds (i.e. divisions of the virtual space), the highest concentration of individuals was found in the landing point in each world, called "Ground Zero". Similar to communicative behaviour associated with a courtyard, individuals would congregate there. We also observed examples of worlds having a meeting place other than the "Ground Zero" location such as in a beach bar, which may also offer specific connotations for interaction.

In the two-dimensional space WBS, rather than providing an embodiment through visual forms, usernames and linked images seemed to provide a degree of embodiment. But we also see differences with respect to the portrayal of space. In contrast to the flat two-dimensional plane, a three-dimensional graphical environment conveys a landscape for interaction. As the user navigates through the space, the environment and artefacts change as one passes by. Also, one can change perspectives, from first person for communication purposes to third person for navigation. Distance perception exists: a small avatar or a small house is far away. Thus, in AW, the three-dimensional metaphor offers certain cues that influence 
users to behave and interact in a virtual space in ways that are different to how people interact with a twodimensional virtual space metaphor.

These similarities to a real environment may translate into navigation playing a stronger role in a threedimensional space for social interaction. In a two-dimensional space, conversation rather than movement is the primary focus. In the three-dimensional space, movement and conversation both play a role and differ in relevance depending on the user's task (e.g. house building vs. group interaction). The visualisation of the three-dimensional space provides a visible awareness of others; a sense of their presence is not only based on conversation but also on their movement and positioning. Each user then maintains a visible location within the three-dimensional world. From a cognitive viewpoint, one can easily grasp the information of who is interacting with whom (within a close distance) simply by observing the clusters of avatars. In a two-dimensional chatroom, gaining this information requires more effort; one must observe the conversational threads. However, this ease of awareness breaks down when the avatars do not change position as they change conversational partners [33].

In a three-dimensional space, proximity of avatars may be a factor in determining who one converses with and whether others may enter a conversation (i.e. by signalling privacy). In a two-dimensional space, conversational partners are not linked by proximity. In addition, the three-dimensional landscape influenced specific behavioural actions (i.e. groups collectively viewing houses or dancing) and often entered into the conversation (e.g. speaking together about the view while suspended in space). In contrast to the more varied landscape, the rooms in WBS are fairly uniform.

In each environment, social conventions involving the use of the space exist. This was observed in AW from the personal distance separating interacting avatars, the reluctance of avatars to invade another's personal space, especially during navigation, the positioning of an avatar in the environment to indicate privacy, and in the adverse reactions towards avatars when their personal spaces were violated. In WBS, the conventions were less obvious, but some references in conversation were made by users concerning navigation and the personal space of other users. In addition, reactions to crowded environments occurred, illustrating the cognitive difficulties of interacting with large numbers of users.

The existence of social conventions may support Harrison and Dourish's [18] notion of place. They consider placeness as an evolved social understanding of the type of behaviour and actions that are appropriate within a space. The architecture and inhabitants may have collectively functioned to enable conventions to emerge. Without the particular concept of place that developed, we would not have expected some of the conventions in AW to exist, such as personal space. Similarly, the theme chatrooms of WBS also appear to promote an idea of place, since conversations appropriate to the theme occurred in them. While both AW and WBS also have conventions that are more of a linguistic nature, such as the use of acronyms, AW appears to have additional conventions associated with an idea of a three-dimensional space.

Although we cannot draw strong conclusions from this exploratory study, we can suggest that such interpersonal behaviours may be influenced by factors such as the presence of an embodiment (avatar), expectations about the space, design features, and the presence of others. But as new forms of shared information spaces continue to emerge, corresponding new notions of placeness and appropriate behavioural conventions will also need to develop. Our basic results that shared virtual environments are regarded as social spaces, with corresponding socially acceptable behaviours, is also consistent with results found from studies of other virtual environments $[1,54,58]$. CVEs are an exciting new form of virtual communication, and we hope that our results can stimulate further research into their behavioural aspects.

\section{Acknowledgements}

We thank Andreas Dieberger, Kaisa Kauppinen, Alan Munro and Mike Robinson for their useful feedback during earlier drafts of this chapter. We thank Deborah Stacey for her generous support at the University of Guelph. 


\section{References}

1.Bruckman A, Resnick M. The MediaMOO Project: Constructionism and Professional Community, Convergence 1995; 1 (1): 94-109. http://asb.www.media.mit.edu/people/asb/convergence.html

2.Neal L. Virtual Classrooms and Communities. In: Hayne SC, Prinz W (eds) Proceedings of ACM Group '97, ACM: New York, 1997, pp 81-90

3.Fahlén LE, Brown CG Stahl O Carlsson C.. A space based model for user interaction in shared synthetic environments, In ACM Conference on Human Factors in Computing (InterCHI '93): ACM Press, New York, 1993

4.Greenhalgh C, Benford S. MASSIVE: A virtual reality system for tele-conferencing. ACM Transactions on Computer Human Interfaces (TOCHI), 1995; 2(3). ACM Press, 239-261

5.Active Worlds: http://www.activeworlds.com

6.Gómez EJ, Quiles JA, Sanz MF, del Pozo F. A user-centered cooperative information system for medical imaging diagnosis. Journal of the American Society for Information Science, 1998; 49(9): 810-816

7.Hindmarsh J, Fraser M, Heath C, Benford S, Greenhalgh C. Fragmented interaction: Establishing mutual orientation in virtual environments. In: Proceedings of CSCW '98, November 14-18, 1998. ACM Press, New York, 1998, pp 217-226

8.Bannon L, Bødker S. Constructing common information spaces. In: Hughes J, Rodden T, Prinz W, Schmidt K. (eds) Proceedings of the 5th European on Computer Supported Cooperative Work (ECSCW '97) Conference. Kluwer Academic Publishers, Dordrecht, 1997, pp 81-96

9.Fuchs L., Poltrock S, Wojcik R. Business value of three-dimensional virtual environments, SIGGROUP Bulletin, ACM Press, New York, 1998; 19(3): pp 25-29

10.Huxor A. An active worlds interface to BSCW, to enhance chance encounters. Proceedings of Collaborative Virtual Environments '98: CVE '98, University of Manchester, UK, 1998, 87-93

11.Benford S, Brown C, Reynard G, Greenhalgh C. Shared spaces: Transportation, artificiality and spatiality. In: Ackerman M. (ed) Proceedings of CSCW '96, ACM Conference on Human Factors in Computing: ACM Press: New York, 1996, pp 77-86

12.Goldate S. The 'Cyberflaneur' -Spaces and places on the internet. Art Monthly Australia, 1997. http://www.geocities.com/Paris/LeftBank/5696/flaneur.htm

13. Maglio P, Matlock T. Constructing social spaces in virtual environments: Metaphors we surf the web by. In: Workshop on Personalised and Social Navigation in Information Space. SICS Technical Report 98:01, K. Höök, A. Munro, D. Benyon, editors, Stockholm, 1998, pp 138-149

14.Maglio P, Matlock T. The conceptual structure of information space. In: Social Navigation of Information Space, A.J. Munro, K. Höök and D. Beynon, editors. London: Springer, 1999, Chapter 9.

15.Dieberger A, Frank A U. A city metaphor for supporting navigation in complex information spaces. Journal of Visual Languages and Computing, 1998; 597-622

16.Dieberger A. On magic features in (spatial) metaphors. SigLink Newsletter, 4(3), 1995, 8-10

17.Dieberger A. Social navigation in populated information spaces: In: Social Navigation of Information Space, A.J. Munro, K. Höök and D. Beynon, editors. London: Springer, 1999, Chapter 3.

18.Harrison S, Dourish P. Re-Place-ing space: The roles of place and space in collaborative systems. In: Ackerman M (ed) Proceedings of CSCW 1996, Nov. 16-20, 1996. ACM Press. Boston, MA, 1996, pp 6776

19.Heath C. Luff P. (1992): Collaboration and Control: Crisis management and multimedia technology in London Underground Line Control Rooms, Computer Supported Cooperative Work (CSCW), An International Journal, 1992; 1: 69-94

20.Osmond H. Function as a basis of psychiatric ward design. Mental Hospitals, (Architectural supplements) 1957; 83: 235-245

21.Tuan Y. Space and place: The perspective of experience. University of Minnesota Press, Minneapolis, Minn., 1977

22.Altman I, Zube, EH. Public places and spaces. New York: Plenum. 1989 
23.McCormick EJ \& Ilgen D. Industrial and Organizational Psychology. ( $8^{\text {th }}$ edition). Prentice-Hall. Englewood Cliffs, NJ, 1987

24.Field RHG, House RJ. Human behaviour in organizations: A Canadian perspective. Prentice-Hall Canada, Scarborough, Canada, 1995

25.Feldman DC. The development and enforcement of group norms. Academy of Management Review, 1984; 9(1), 47-53

26.Vecchio RP. Organizational behavior. ( $2^{\text {nd }}$ Edition). Dryden, Orlando, FL, 1991

27.Goodman PS, Ravlin E, Schminke M. Understanding groups in organizations. In: Cummings LL, Staw BM (eds) Research in Organizational Behavior 1987, 9, pp 121-173

28.Carroll JM, Thomas JC. Metaphors and the cognitive representation of computing systems. IEEE Transactions on System, Man, And Cybernetics 1982; SMC-12 (2)

29.Dix AJ, Finlay JE, Abowd GD, Beale R. Human-computer interaction (2 ${ }^{\text {nd }}$ Edition). Prentice Hall Europe, Hertfordshire, UK, 1998

30.Bruckman A. Identity Workshop: Emergent Social and Psychological Phenomena in Text-Based Virtual Reality. 1992. ftp:media.mit.edu:/pub/asb/papers/identity-workshop.ps

31.Raybourn E. An intercultural computer-based multi-user simulation supporting participant exploration of identity and power in a text-based networked virtual reality: DomeCity ${ }^{\mathrm{TM}}$ MOO. Unpublished dissertation. Department of Communication, University of New Mexico. Albuquerque, NM, 1998

32.Baym N. The performance of humor in computer-mediated communication. Journal of ComputerMediated Communication 1(2) (online). 1995. http://shum.cc.huji.ac.il/jcmc/vol1/issue2/baym.html

33.Becker B, Mark G. (1998). Constructing social systems through computer-mediated communication. Forthcoming in Virtual Reality

34.Jeffrey P, Mark G. Constructing social spaces in virtual environments: A study of navigation and interaction In: Höök, K, Munro A Benyon D. (eds) Workshop on Personalised and Social Navigation in Information Space SICS Technical Report 98:01, Stockholm, 1998, pp. 24-38

35.Kauppinen K, Kivimäki A, Era T, Robinson M. Producing identity in collaborative virtual environments. In VRST'98: Symposium on Virtual Reality Software and Technology. ACM Press, New York, 1998, 35-42

36.Hall ET. The hidden dimension. Anchor Books. New York, 1966

37.Aiello JR. Human spatial behavior. In: Stokols D, Altman I (eds) Handbook of Environmental Psychology: Volume 1, Wiley \& Sons, New York, 1987, 389-504

38.Knapp ML. Nonverbal communication in human interaction. Holt, New York, 1978

39.Altman I, Vinsel AM. Personal space: An analysis of E.T. Hall's proxemics framework. In: I. Altman \& J.F. Wohlwill (eds) Human behaviour and the environment: Vol. 2. Advances in theory and research. Plenum, New York, 1977, pp 181-259

40.Altman I. The environment and social behavior. Wadsworth, Monterey, 1975

41.Westin A. Privacy and freedom. Atheneum, NewYork, 1970

42.Stokols D. On the distinction between density and crowding: Some implications for future research. Psychological Review 1972; 79(3): 275-278

43.Freedman JL, Heshka S, Levy A. Crowding as an intensifier of the effect of success and failure. (abstract), J.L. Freedman, Crowding and behavior. Freeman, San Francisco, 1975, 151-152

44.Stokols D, Resnick SM. The generalization of residential crowding experiences to non-residential settings. Annual Conference of the Environmental Design Research Association, Lawrence, Kansas, 1975

45.Strickland D, Hodges L, North M, Weghorst S. Overcoming phobias by virtual exposure. Communications of the ACM 1997; 40(8): 34-39

46.Benford S, Greenhalgh C, Lloyd D. Crowded colloborative virtual environments. In: Proceedings of CHI '97, ACM Press, New York, 1997, pp 59-66

47.Citera M. Distributed Teamwork: The impact of communication media on influence and decision quality. Journal of the American Society for Information Science 1998; 49(9): 792-800 
48.Okada K, Maeda F, Ichikawaa Y, Matsushita Y. Multiparty videoconferencing at virtual social distance: MAJIC Design. In: Proceedings of CHI '94, ACM Press, New York, 1994, pp 385-393.

49.Persson P. Towards a psychological theory of close-ups: Experiencing intimacy and threat. KINEMA: A Journal of History, Theory and Aesthetics of Film and Audiovisual Media. University of Waterloo Press, Waterloo, Canada, 1998

50.Meyrowitz J. Television and interpersonal behaviour: Codes of perception and response. In Gumpert G Cathcart R (eds.). Inter/Media: Interpersonal Communication in a Media World. New York, 1986

51.Dieberger A. Personal communication, 1999

52.Fletcher A. The rhetoric of synthetic vs analytic truth on the internet as it relates to nonverbal communication, 1997. http://www.geocities.com/Paris/Metro/1022/nonverbl.htm

53.Masterson J. Nonverbal Communication in Text Based Virtual Realities. M.A. Thesis. University of Montana. 1996. http://www.montana.com/john/thesis/

54.Turkle S. Life on the screen: Identity in the Age of the Internet. Touchstone, New York, 1997

55.Nilan M. Ease of user navigation through digital information spaces. Proceedings of the 37 Allerton Institute 1995, Monticello, Illinois, (on-line), 1995. http://edfu.lis.uiuc.edu/allerton/95/s4/nilan.html

56.Munro A. (1998). Inhabiting information space: Work artefacts and new realities. In: Dahlbaeck N (ed.). Exploring Navigation; Towards a Framework for Design and Evaluation of Navigation in Electronic Spaces. Stockholm: SICS Technical Report, T98:01, 91-114

57.Waterworth JA. Personal spaces: three-dimensional spatial worlds for information exploration, organisation and communication. In: Earnshaw R, Vince J (eds.): The Internet in three-dimensional: Information, Images, and Interaction. Academic, San Diego, CA, 1997. http://www.informatik.umu.se/\%7ejwworth/spaces.pdf

58.Curtis P. MUDding: Social phenomena in text-based virtual realities. In: Ludlow P (ed) High noon on the electronic frontier: Conceptual issues in cyberspace. MIT Press, Cambridge, Mass, 1996, pp 347-373 